\title{
COMMISSIONING OF THE TRIUMF ISAC RF SYSTEM
}

\author{
K. Fong, S. Fang, M. Laverty, J. Lu, R. L. Poirier, TRIUMF, Vancouver, Canada V6T 2A3
}

\begin{abstract}
The ISAC RF system at present consists of a Radio Frequency Quadrupole accelerator, five Drift Tube Linear Accelerators, six bunchers, two choppers and a bunch rotator. The RFQ operates at the fundamental frequency of $35.36 \mathrm{MHz}$, while the DTLs operate at the third harmonic frequency of $106.08 \mathrm{MHz}$. The operating power ranges from $45 \mathrm{~W}$ to $120 \mathrm{~W}$ for the choppers, $1 \mathrm{~kW}$ to 20 $\mathrm{kW}$ for the DTLs and bunchers, and $80 \mathrm{~kW}$ for the RFQ. These cavities have been commissioned to operate synchronously with both closed-loop amplitude and phase regulation, as well as automatic tuning of the cavities. This paper gives a brief summary of the commissioning experience.
\end{abstract}

\section{INTRODUCTION}

Figure 1 shows all the RF cavities along the beamline in ISAC. The ISAC RFQ and the prebuncher were commissioned in 1998. The commissioning process was paused for beam dynamic studies. It was resumed in mid 1999, when the rebuncher, first DTL tank and the first buncher were commissioned[1]. There was another brief pause in the commissioning process while beam dynamic studies were being performed on the DTL. Then in late 2000 and early 2001 the remaining four DTL tanks and 4 bunchers were commissioned, as well as two choppers and a bunch rotator. The bunchers, choppers, bunch rotator and DTLs operate at various sub-harmonics and harmonics of the RFQ frequency. The various operating frequencies includes 5.89, 11.79, 23.57, 35.36, 106.08 MHz. The master frequency source is a direct digital synthesiser with temperature stabililzed crystal oscillator reference. Various phase locked loops and harmonic multipliers are used to synthesis the required harmonics and subharmonics. Solid state amplifiers are used to power cavities that are less than $2 \mathrm{~kW}$ in power requirement, and vacuum tube amplifiers are used for cavities that require higher power than $2 \mathrm{~kW}$. Each cavity is individually regulated and tuned using two digital signal processors. Table 1 summarizes the geometry and power requirements for all the RF cavities.

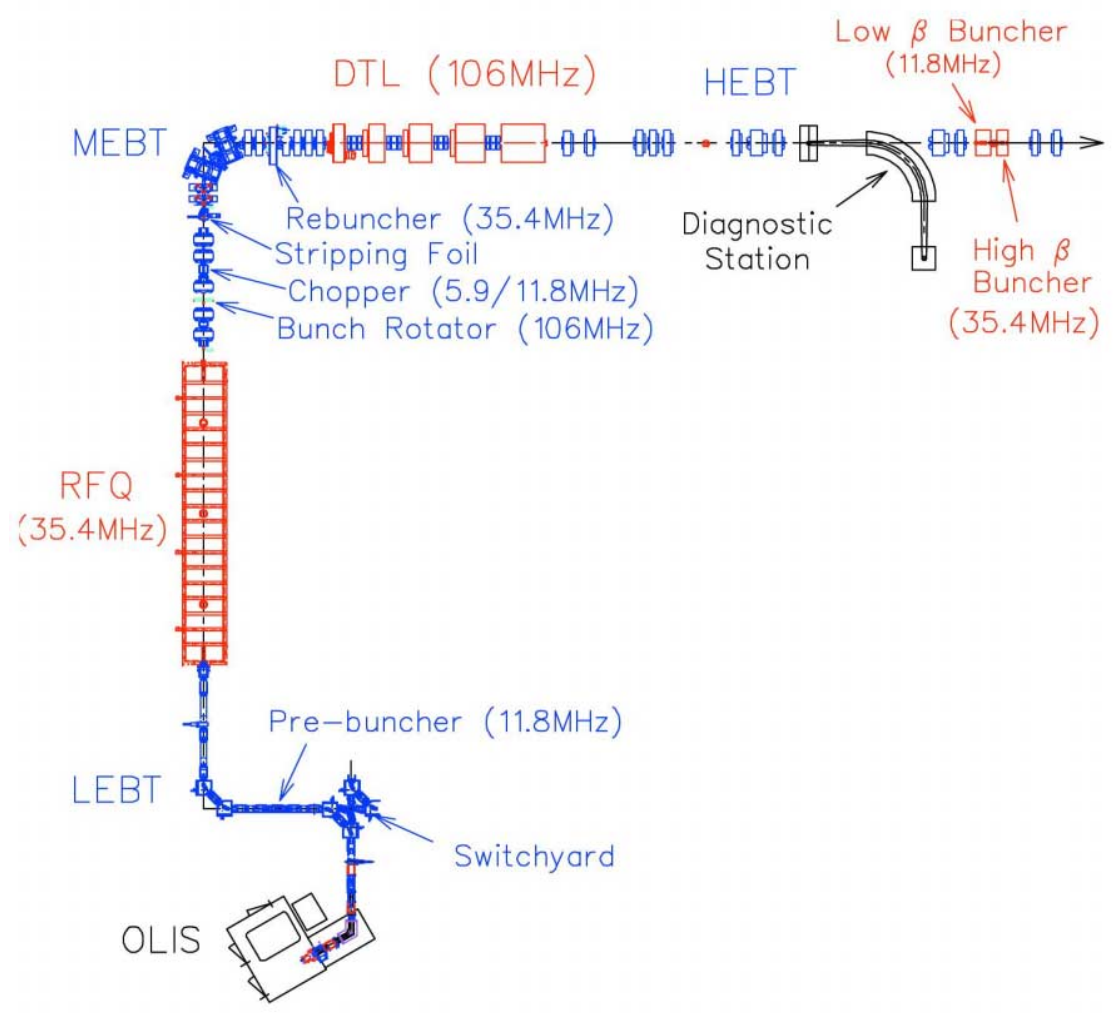

Figure 1: RF cavities in ISAC 


\section{PHASE ADJUSTMENTS}

Since the impedance at the input of the cavity is mainly capacitive or inductive except at the resonant frequency, high impedance occurs at the input of the transmission line at regular intervals in frequency due to the transformation effect of the transmission line. If the amplifier gain is sufficiently high at these frequencies, oscillations can develop from parasitic voltage feedback. For stable amplifier operation the length of the transmission line between the final amplifier and the cavity must be adjusted properly so that these frequencies falls outside of the passband of the amplifier. In the RFQ, a transmission line trombone is used to adjust the length of the transmission line. In all other cavities, their transmission lines are trimmed manually to achieve the desired lengths.

Table 1: RF cavities parameters

\begin{tabular}{|c|c|c|c|}
\hline Device & Structure & $\begin{array}{l}\text { Operating } \\
\text { Frequency }\end{array}$ & $\begin{array}{l}\text { Nominal } \\
\text { Power }\end{array}$ \\
\hline Prebuncher & $\begin{array}{l}\text { Parallel Plate } \\
+\mathrm{R}\end{array}$ & $\begin{array}{c}11,23,35 \\
\mathrm{MHz}\end{array}$ & $500 \mathrm{~W}$ \\
\hline RFQ & & $35 \mathrm{MHz}$ & $80 \mathrm{~kW}$ \\
\hline $\begin{array}{l}5 \mathrm{MHz} \\
\text { chopper }\end{array}$ & $\begin{array}{l}\text { Parallel Plate } \\
+ \text { Coil }\end{array}$ & $5 \mathrm{MHz}$ & $45 \mathrm{~W}$ \\
\hline $\begin{array}{l}11 \mathrm{MHz} \\
\text { chopper }\end{array}$ & $\begin{array}{l}\text { Parallel Plate } \\
+ \text { Coil }\end{array}$ & $11 \mathrm{MHz}$ & $120 \mathrm{~W}$ \\
\hline $\begin{array}{l}\text { Bunch } \\
\text { Rotator }\end{array}$ & Split Ring & $35 \mathrm{MHz}$ & $3 \mathrm{~kW}$ \\
\hline Rebuncher & Spiral & $106 \mathrm{MHz}$ & $1 \mathrm{~kW}$ \\
\hline DTL1 & $\mathrm{IH}$ & $106 \mathrm{MHz}$ & $3.9 \mathrm{~kW}$ \\
\hline Buncher 1 & Split Ring & $106 \mathrm{MHz}$ & $8 \mathrm{~kW}$ \\
\hline DTL2 & $\mathrm{IH}$ & $106 \mathrm{MHz}$ & $10 \mathrm{~kW}$ \\
\hline Buncher2 & Split Ring & $106 \mathrm{MHz}$ & $10.2 \mathrm{~kW}$ \\
\hline DTL3 & $\mathrm{IH}$ & $106 \mathrm{MHz}$ & $16 \mathrm{~kW}$ \\
\hline Buncher3 & Split Ring & $106 \mathrm{MHz}$ & $11.6 \mathrm{~kW}$ \\
\hline DTL4 & $\mathrm{IH}$ & $106 \mathrm{MHz}$ & $19 \mathrm{~kW}$ \\
\hline DTL5 & $\mathrm{IH}$ & $106 \mathrm{MHz}$ & $20.3 \mathrm{~kW}$ \\
\hline $\begin{array}{l}\text { High } \beta \\
\text { Buncher }\end{array}$ & Spiral & $35 \mathrm{MHz}$ & $12 \mathrm{~kW}$ \\
\hline $\begin{array}{l}\text { Low } \beta \\
\text { Buncher }\end{array}$ & $\begin{array}{l}\text { Parallel Plate } \\
+ \text { Coil }\end{array}$ & $11 \mathrm{MHz}$ & $1.5 \mathrm{~kW}$ \\
\hline
\end{tabular}

The design of the RF control system[2,3] requires that for proper operation the RF phase from the modulator to the demodulator must be an integer multiple of $360^{\circ}$. This condition is necessary for minimum VSWR when operated under self-excitation. When operated in driven mode, this condition is necessary for the proper operation of the tuner when operated under driven frequency, and for minimum cross talk between the In-Phase and Quadrature-Phase regulating loops. The design of the RF control system also makes the initial adjustment of this phase quite easy. The control system is built such that the reference signals at both the modulator and the demodulator have the same phase. Low level RF pulses are injected into the cavity and the tuner is moved to tune the cavity resonant frequency to the driven frequency by peaking the amplitude. The RF is switched to $\mathrm{CW}$ and the loop phase shifter is adjusted such that the input phase equals the output phase. Several iterations between adjustment of the tuner and the phase shifter may be necessary. This ensures that the RF phase around the loop is an integer multiple of $360^{\circ}$ when the cavity is at resonance.

\section{RF CONDITIONING}

A new cavity or a cavity that has been idle for some times has micro protrusions and contaminants on its RF surface. These imperfections can produce field emissions, which in turn cause multipactoring, sparking and electron beam loading. Any of these can prevent the cavity from reaching its designed operating voltage and can cause damage to the cavity. RF conditioning uses RF power to cleanse the cavity and rid the surfaces of these imperfections. Each of the ISAC cavities required extensive RF conditioning. We have employed two methods to RF condition a cavity. The first method applies CW RF power to a cavity, the power and the rate at which it rises being limited by the vacuum in the cavity. Because of its $\mathrm{CW}$ nature, this method is very efficient in cleaning an RF surface that contains many contaminants. However, at high power level this method can cause overheating of the transmission line and the coupling loop. Its operation should therefore be limited to medium power level. The second method subjects the cavity to high power, short duration RF pulses. This involves pulsing the cavity with a pulse of $100 \mu$ s or less, but with a peak RF power of the order of $140 \%$ of the peak designed power. This method is useful to remove the contaminants that act as field emitters only at high RF surface field while keeping the average RF power to an acceptable level.

Experience has shown that the first method is better in a brand new cavity in order to reach a mid level RF voltage. Vacuum was typically in the high $10^{-7}$ torr range at the start of conditioning. During conditioning the vacuum was keep below $6 \times 10^{-6}$ torr by limiting the RF input power. This phase of conditioning is completed when the vacuum reaches a stable value in the low $10^{-6}$ torr range when medium power is applied to the cavity. For new DTL cavities this process typically took several hours. Longer times were required for the higher power bunchers.

The second method is then used to clean the cavity for stable operation at high RF power. Typically the pulse width is $32 \mu \mathrm{s}$ and the repetition rate is $2000 \mathrm{~Hz}$. About 10 to 15 minutes of pulsing is all that is required. The cavity voltage and the vacuum are continuously monitored during this period. The cavity voltage can be seen to intermittently collapse but recover in the subsequent pulse, with corresponding pressure pulses observed in the vacuum gauge. Again the vacuum is kept below $6 \times 10^{-6}$ torr by limiting the RF input power. We were able to observe the electrodes of the RFQ through its cavity's glass porthole during this conditioning. Flashing bright spots were seen at the electrode surfaces, gradually diminishing in frequency as the cavity was conditioned. 
The cavity is considered conditioned when the cavity voltage is no longer observed to collapse.

\section{POWER UP SEQUENCING}

After a cavity has been conditioned, it can then be powered up to its operating voltage. The sequence of events are illustrated in Figure 2. As the resonant frequencies of the RF cavities change when the temperature of the cavities are different, powering up a cavity requires the tuner be moved to a preset cold start position. Pulsed RF with an increasing amplitude is applied to the cavity. When the amplitude of the RF in the cavity reaches a preset value above the multipactoring threshold, the pulsed RF is switched to CW. The tuning loop is activated after waiting for a few seconds for the voltage levels to stabilize. The input power continues to increase until the cavity voltage exceeds the setpoint value, at which point the voltage feedback loops can be closed smoothly without generating a transient. The input power is now controlled by the voltage feedback loops, but its limit continues to rise to a preset value to provide headroom for the voltage regulation, but prevent uncontrolled dumping of power in case of fault. This process is first performed manually to extract all the necessary preset values, and subsequently can be performed automatically through the control system.

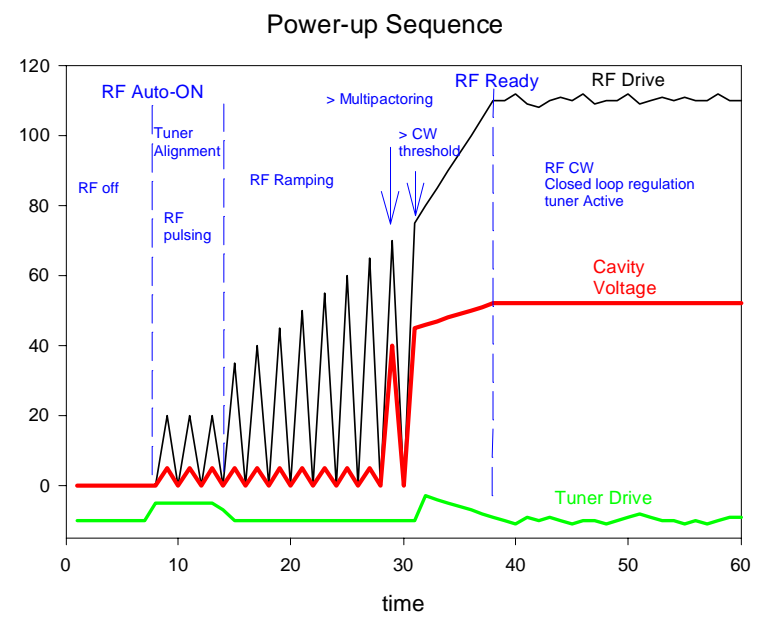

Figure 2: Timing in Automatic Power-up Sequence

In the separated function DTL section, the bunchers[4] do not have vacuum pumps, but are pumped through their attached DTL tanks. The high pumping resistance requires more care in powering up the bunchers than the DTLs.

\section{RELIABLE OPERATION}

Since the accelerator consists of 15 separate RF cavities, it is very important to achieve high reliability for each of these cavities. During the initial phase of commissioning, a lot of problems were encountered while trying to attain reliable operation.
Ionization vacuum gauges were installed at all cavities to monitor the residue pressures of the cavities. These gauges, however, are sensitive to RF interference and caused spurious vacuum readings. The solution was to install extension tubes and extra shields to reduce the RF level at the sensor.

Amplifiers that are rated over $10 \mathrm{~kW}$ use tetrodes as the final amplifiers. Their screen power supplies were particularly problematic. These were commercial switching regulators and their designs were prone to RF interference and were unable to handle transient loads. These had resulted in many spurious trips of the screen power supplies. 3 out of 9 of these power supplies have been replaced with unregulated ones that are more immune to RF interference. We are presently in the process of replacing the remaining 5 power supplies.

DTL tank 3 was the first cavity that requires a nominal power exceeding $12 \mathrm{~kW}$. It has a stainless steel tank with an inside surface of electroplated copper. During the initial testing phase it was discovered that the measured Q was substantially lower than the calculated Q. As a result additional cooling water channels were added to the outside surface to keep the tank temperature below $45^{\circ} \mathrm{C}$. This delayed the installation of the tank in the beam line and the tank was only baked out for a short duration. During the commissioning of this tank, we found that the cavity load increased rapidly and the tuning deteriorated when the input RF power exceeded $12 \mathrm{~kW}$. We did not, however, observe a similar deterioration in vacuum. After baking out this cavity at $55^{\circ} \mathrm{C}$ for overnight this extraneous load disappeared and we were able to power the cavity to nominal voltage using full power.

\section{SUMMARY}

The 15 cavities in the ISAC accelerator have been commissioned in a relatively short time. The earlier cavities took three weeks to commission. As we have gained experience during the commissioning, the time required to commission the latest cavity was less than a week. Their reliability has increased as we have gained experience in operating them.

\section{REFERENCES}

[1] R. L. Poirier et al, "RF Systems of the TRIUMF ISAC Facility," Proc. IEEE 1999 Part. Accel. Conf., New York, (1999).

[2] K. Fong, M. Laverty and S. Fang, "RF Control Systems for the TRIUMF ISAC RF Structures," This Proceeding.

[3] M. Laverty, K. Fong and S. Fang, "Design and Testing of the ISAC RFQ Control System", Proc. IEEE 1999 Part. Accel. Conf., New York, (1999).

[4] Y. V. Bylinsky et al, "High Power Test of the ISAC Triple Gap Buncher Operating in CW Mode," Proc. IEEE 1999 Part. Accel. Conf., New York, (1999). 